15

ournal of

Mechanics of

Materials and Structures

0.

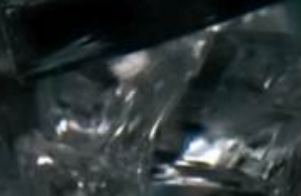

$+8$

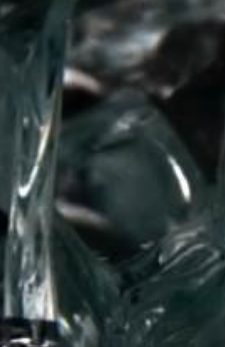

की

(S)
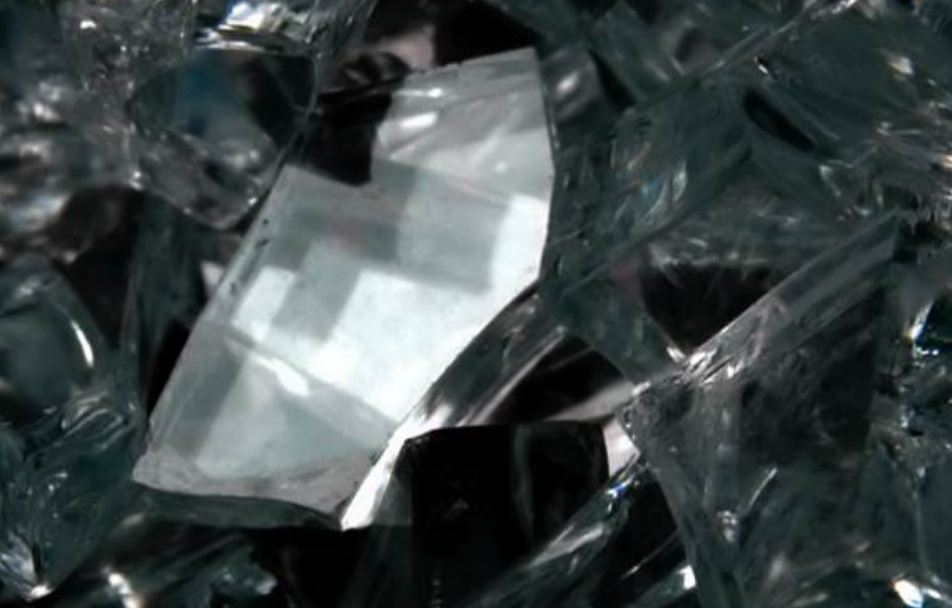

PISPERSION OF GUIDED WAVES IN INITIALLY STRESSED LAYERED PLATÉS 25

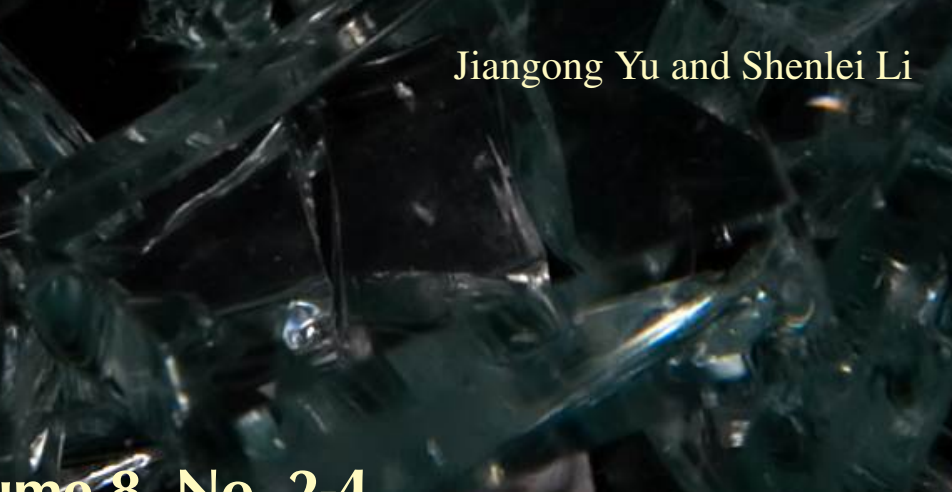

Volume 8, No. 2-4,

- April-June 2013

N.

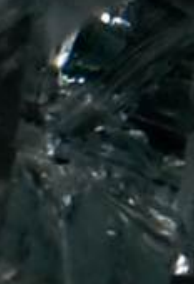




\title{
DISPERSION OF GUIDED WAVES IN INITIALLY STRESSED LAYERED PLATES
}

\author{
JIANGONG YU AND SHENLEI Li
}

\begin{abstract}
Based on the theory of the mechanics of incremental deformations, guided wave propagation in multilayered plates under initial stresses in the thickness and wave-propagation directions is investigated. The Legendre polynomial series method is used to solve the coupled wave equations and its convergence is discussed along with numerical examples. The effects of initial stresses on Lamb-like waves and SH waves are analyzed, and numerical results show that they are quite distinct. The frequency variation is not linear with the increase of the initial stresses in both directions. The effects of the initial stress in the thickness direction are very different from those in the longitudinal direction.
\end{abstract}

\section{Introduction}

Because of the limitations of manufacturing and assembling technology, initial stresses in structural elements, especially in layered structures, are inevitable. The working loads on structures can be taken as the initial stresses. Thus, the problem of initially stressed structures is very common and it is very important to study it in both the practical and the theoretical sense.

Wave propagation in structures with initial stresses has received considerable attention. Chen and Wright [1966] derived the frequency equations for wave propagation in an initially stressed circular cylinder. Murdoch [1977] considered the effect of interfacial stress on the propagation of Stoneley waves. Ogden and Sotiropoulos [1995] studied interfacial waves in prestressed layered incompressible elastic solids. Gei [2008] investigated small-amplitude interfacial waves along a thin film between two prestressed, incompressible elastic media. Rayleigh waves in a magnetoelastic initially stressed conducting medium with a gravity field were investigated in [El-Naggar et al. 1994]. Abd-Alla and Ahmed [1999] investigated Love wave propagation in a nonhomogeneous orthotropic elastic layer with initial stress overlying a semiinfinite medium. Abd-Alla [1999] presented the effect of initial stress on axisymmetric waves in an orthotropic hollow cylinder. Qian et al. [2004] studied the effect of initial stress on Love waves in an elastic substrate with a piezoelectric layer and a piezoelectric substrate with an elastic layer. Du et al. [2007] studied Love wave propagation in layered magnetoelectroelastic structures with initial stress. Garg [2007] considered the effect of initial stress on harmonic plane waves in viscoelastic anisotropic media. Gupta et al. [2012] investigated torsional surface waves in an initially stressed heterogeneous half-space covered by a homogeneous layer. Bigoni et al. [2008] studied the filtering and band gap characteristics of a prestressed, stiff layer on an elastic half-space.

The above investigations are all about surface waves, subsurface waves, and interfacial waves. Guided wave propagation in structures with initial stresses has also received attention. Akbarov and Guliev [2009] studied axisymmetric longitudinal waves in a prestrained compound circular cylinder. Akbarov et al. [2011b] studied the case of torsional waves. Akbarov and Guliev [2010] investigated axisymmetric

Keywords: multilayered plate, guided waves, initial stress, orthogonal polynomial, dispersion curves. 
longitudinal waves in a prestrained circular cylinder embedded in a prestrained compressible infinite medium. These three papers are all about circular cylinders. As they are more common structures, initially stressed multilayered plates have received more attention. Kayestha et al. [2010] obtained dispersion curves for a perfectly bonded prestressed compressible elastic bilayered plate. Wijeyewickrema and Leungvichcharoen [2009] investigated the influence of imperfections in the contact conditions between the layers of a prestrained three-layered plate. These two investigations do not allow readers to draw any concrete conclusions about the influence of the initial strains on the wave propagation velocity or on wave dispersion in layered media. Akbarov et al. [2008; 2011a] and Zamanov and Agasiyev [2011] investigated these issues. Akbarov et al. [2008] and Zamanov and Agasiyev [2011] studied the influence of initial strains in the wave-propagation direction on the Lamb wave dispersion curves. Akbarov et al. [2011a] studied the influence of initial strains in the thickness direction on the Lamb wave dispersion curves. Furthermore, small-amplitude waves in prestressed [Rogerson and Sandiford 1996; Gei et al. 2004; Gei 2008] and large deformed [Shmuel et al. 2012] multilayered structures have been given attention.

This paper simultaneously investigates the influence of initial stresses in two directions, the thickness and wave-propagation directions. Their different effects are discussed. We also illustrate the case of SH waves. The influences on Lamb waves and on SH waves are very different.

\section{Mathematics and formulation of the problem}

Consider an orthotropic $N$-layered plate with initial stresses in two directions, $S_{x x}=-\sum_{n=1}^{N} P_{n} \pi_{h_{n-1}, h_{n}}(z)$ and $S_{z z}=-Q$, as shown in Figure 1. The rectangular window function is

$$
\pi_{h_{n-1}, h_{n}}(z)= \begin{cases}1, & h_{n-1} \leq z \leq h_{n}, \\ 0, & \text { elsewhere }\end{cases}
$$

The plate is infinite in the horizontal direction and has a total thickness $h_{N}$. We place the horizontal $(x, y)$-plane of a Cartesian coordinate system on the bottom surface and let the plate be in the positive $z$ region, where the medium occupies the region $0 \leq z \leq h_{N}$.

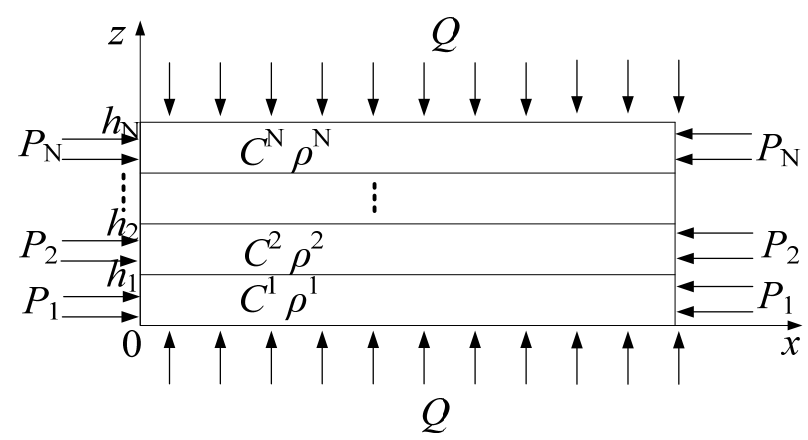

Figure 1. Schematic diagram of a layered plate under initial stresses. 
According to [Biot 1965] (see also [Selim and Ahmed 2006]) the dynamic equations for the plate with initial stresses are governed by

$$
\begin{array}{r}
\frac{\partial T_{x x}}{\partial x}+\frac{\partial T_{x y}}{\partial y}+\frac{\partial T_{x z}}{\partial z}-\frac{1}{2}\left(\frac{\partial u_{x}}{\partial z}+\frac{\partial u_{z}}{\partial x}\right) \frac{\partial S_{x x}}{\partial z}+S_{x x} \frac{\partial \omega_{z}}{\partial y}+\left(S_{z z}-S_{x x}\right) \frac{\partial \omega_{y}}{\partial z}=\rho \frac{\partial^{2} u_{x}}{\partial t^{2}} \\
\frac{\partial T_{x y}}{\partial x}+\frac{\partial T_{y y}}{\partial y}+\frac{\partial T_{y z}}{\partial z}-S_{z z} \frac{\partial \omega_{x}}{\partial z}+S_{x x} \frac{\partial \omega_{z}}{\partial x}=\rho \frac{\partial^{2} u_{y}}{\partial t^{2}} \\
\frac{\partial T_{x z}}{\partial x}+\frac{\partial T_{y z}}{\partial y}+\frac{\partial T_{z z}}{\partial z}+\left(S_{z z}-S_{x x}\right) \frac{\partial \omega_{y}}{\partial x}-S_{z z} \frac{\partial \omega_{x}}{\partial y}=\rho \frac{\partial^{2} u_{z}}{\partial t^{2}}
\end{array}
$$

where

$$
\omega_{x}=\frac{1}{2}\left(\frac{\partial u_{z}}{\partial y}-\frac{\partial u_{y}}{\partial z}\right), \quad \omega_{y}=\frac{1}{2}\left(\frac{\partial u_{x}}{\partial z}-\frac{\partial u_{z}}{\partial x}\right), \quad \omega_{z}=\frac{1}{2}\left(\frac{\partial u_{y}}{\partial x}-\frac{\partial u_{x}}{\partial y}\right) .
$$

The relationships between the strain and displacement components can be expressed as

$$
\begin{aligned}
& \epsilon_{x x}=\frac{\partial u_{x}}{\partial x}, \quad \epsilon_{y y}=\frac{\partial u_{y}}{\partial y}, \quad \epsilon_{z z}=\frac{\partial u_{z}}{\partial z}, \\
& \epsilon_{y z}=\frac{1}{2}\left(\frac{\partial u_{y}}{\partial z}+\frac{\partial u_{z}}{\partial y}\right), \quad \epsilon_{x z}=\frac{1}{2}\left(\frac{\partial u_{x}}{\partial z}+\frac{\partial u_{z}}{\partial x}\right), \quad \epsilon_{x y}=\frac{1}{2}\left(\frac{\partial u_{x}}{\partial y}+\frac{\partial u_{y}}{\partial x}\right) .
\end{aligned}
$$

Here $u_{i}, T_{i j}$ and $\epsilon_{i j}$ are the elastic displacements, stresses, and strains, respectively, while $\rho$ is the density of the plate.

By introducing the rectangular window function $\pi_{0, h_{N}}(z)$, the stress-free boundary $\left(T_{z z}=T_{x z}=T_{y z}=0\right.$ at $z=0, z=h_{N}$ ) is automatically incorporated in the constitutive relations of the plate [Datta and Hunsinger 1978]

$$
\begin{aligned}
T_{x x} & =C_{11} \epsilon_{x x}+C_{12} \epsilon_{y y}+C_{13} \epsilon_{z z}, & T_{y y} & =C_{12} \epsilon_{x x}+C_{22} \epsilon_{y y}+C_{23} \epsilon_{z z}, \\
T_{z z} & =\left(C_{13} \epsilon_{x x}+C_{23} \epsilon_{y y}+C_{33} \epsilon_{z z}\right) \pi_{0, h_{N}}(z), & T_{y z} & =2 C_{44} \epsilon_{y z} \pi_{0, h_{N}}(z), \\
T_{x z} & =2 C_{55} \epsilon_{x z} \pi_{0, h_{N}}(z), & T_{x y} & =2 C_{66} \epsilon_{x y},
\end{aligned}
$$

where $C_{i j}$ are the elastic coefficients.

For the layered plate these coefficients are expressed as

$$
C_{i j}=\sum_{n=1}^{N} C_{i j}^{n} \pi_{h_{n-1}, h_{n}}(z)
$$

where $N$ is the number of the layers and $C_{i j}^{n}$ is the elastic constant of the $N$-th material. Similarly, the mass density can be expressed as

$$
\rho=\sum_{n=1}^{N} \rho^{n} \pi_{h_{n-1}, h_{n}}(z)
$$


For a free harmonic wave being propagated in the $x$ direction in a plate, we assume the displacement components to be of the form

$$
\begin{aligned}
& u_{x}(x, y, z, t)=\exp (i k x-i \omega t) U(z), \\
& u_{y}(x, y, z, t)=\exp (i k x-i \omega t) V(z), \\
& u_{z}(x, y, z, t)=\exp (i k x-i \omega t) W(z) .
\end{aligned}
$$

$U(z), V(z)$, and $W(z)$ represent the amplitude of vibration in the $x, y$, and $z$ directions, respectively, while $k$ is the magnitude of the wave vector in the propagation direction and $\omega$ is the angular frequency.

Substituting (3)-(6) into (1), the governing differential equations in terms of displacement components can be obtained:

$$
\begin{aligned}
& U^{\prime \prime} \sum_{n=1}^{N}\left(C_{55}^{n}+0.5 P_{n}-0.5 Q\right) \pi_{h_{n-1}, h_{n}}(z)+U^{\prime}\left(\sum_{n=1}^{N}\left(C_{55}^{n}+0.5 P_{n}\right) \pi_{h_{n-1}, h_{n}}(z)\right)^{\prime} \\
& -k^{2} U \sum_{n=1}^{N}\left(C_{11}^{n}+P_{n}\right) \pi_{h_{n-1}, h_{n}}(z)+i k W^{\prime} \sum_{n=1}^{N}\left(C_{13}^{n}+C_{55}^{n}+0.5 P_{n}+0.5 Q\right) \pi_{h_{n-1}, h_{n}}(z) \\
& +i k W\left(\sum_{n=1}^{N}\left(C_{55}^{n}+0.5 P_{n}\right) \pi_{h_{n-1}, h_{n}}(z)\right)^{\prime}=-\omega^{2} U \sum_{n=1}^{N} \rho^{n} \pi_{h_{n-1}, h_{n}}(z),
\end{aligned}
$$

$$
\begin{array}{r}
V^{\prime \prime} \sum_{n=1}^{N}\left(C_{44}^{n}-0.5 Q\right) \pi_{h_{n-1}, h_{n}}(z)+V^{\prime}\left(\sum_{n=1}^{N} C_{44}^{n} \pi_{h_{n-1}, h_{n}}(z)\right)^{\prime}-k^{2} V \sum_{n=1}^{N}\left(C_{66}^{n}-0.5 P_{n}\right) \pi_{h_{n-1}, h_{n}}(z) \\
=-\omega^{2} V \sum_{n=1}^{N} \rho^{n} \pi_{h_{n-1}, h_{n}}(z)
\end{array}
$$

$$
\begin{aligned}
W^{\prime \prime} \sum_{n=1}^{N}\left(C_{33}^{n}+Q\right) \pi_{h_{n-1}, h_{n}}(z)+i k U^{\prime} \sum_{n=1}^{N}\left(C_{13}^{n}+C_{55}^{n}+0.5 P_{n}+0.5 Q\right) \pi_{h_{n-1}, h_{n}}(z) \\
-k^{2} W \sum_{n=1}^{N}\left(C_{55}^{n}-0.5 P_{n}+0.5 Q\right) \pi_{h_{n-1}, h_{n}}(z)+W^{\prime}\left(\sum_{n=1}^{N}\left(C_{33}^{n}+Q\right) \pi_{h_{n-1}, h_{n}}(z)\right)^{\prime} \\
+i k U\left(\sum_{n=1}^{N}\left(C_{13}^{n}+Q\right) \pi_{h_{n-1}, h_{n}}(z)\right)^{\prime}=-\omega^{2} W \sum_{n=1}^{N} \rho^{n} \pi_{h_{n-1}, h_{n}}(z)
\end{aligned}
$$

where the superscript ( $)^{\prime}$ is the partial derivative for $z$. Obviously, (7b) is independent of the other two equations. It represents the antiplane SH waves. The other two, (7a) and (7c), control the in-plane Lamb-like waves.

To solve the coupled wave (7), we expand the field quantities of each layer into one specific Legendre orthogonal polynomial series:

- In the first layer

$$
u_{a}^{1}=\sum_{m=0}^{\infty} p_{m, 1}^{a} Q_{m}^{1}(z) \exp (i k x)
$$


with

$$
Q_{m}^{1}(z)=\sqrt{\frac{2 m+1}{h_{1}}} L_{m}\left(\frac{2}{h_{1}} z-1\right)
$$

and

$$
u_{a}^{1}\left(z=h_{1}\right)=u_{a}^{1, h_{1}}=\sum_{m=0}^{\infty} p_{m, 1}^{a} Q_{m}^{1}\left(z=h_{1}\right) \exp (i k x)
$$

- In the second layer

$$
u_{a}^{2}=u_{a}^{1, h_{1}}+\left(z-h_{1}\right) \sum_{m=0}^{\infty} p_{m, 2}^{a} Q_{m}^{2}(z) \exp (i k x)
$$

with

$$
Q_{m}^{2}(z)=\sqrt{\frac{2 m+1}{h_{2}-h_{1}}} L_{m}\left(\frac{2}{h_{2}-h_{1}} z-\frac{h_{2}+h_{1}}{h_{2}-h_{1}}\right)
$$

and

$$
u_{a}^{2}\left(z=h_{2}\right)=u_{a}^{2, h_{2}}=u_{a}^{1, h_{1}}+\left(h_{2}-h_{1}\right) \sum_{m=0}^{\infty} p_{m, 2}^{a} Q_{m}^{2}\left(z=h_{2}\right) \exp (i k x) .
$$

- The subsequent layers follow as such.

- In the last layer

$$
u_{a}^{N}=u_{a}^{N-1, h_{N-1}}+\left(z-h_{N-1}\right) \sum_{m=0}^{\infty} p_{m, N}^{a} Q_{m}^{N}(z) \exp (i k x)
$$

with

$$
Q_{m}^{N}(z)=\sqrt{\frac{2 m+1}{h_{N}-h_{N-1}}} L_{m}\left(\frac{2}{h_{N}-h_{N-1}} z-\frac{h_{N}+h_{N-1}}{h_{N}-h_{N-1}}\right)
$$

and

$$
u_{a}^{N}\left(z=h_{N}\right)=u_{a}^{N, h_{N}}=u_{a}^{N-1, h_{N-1}}+\left(h_{N}-h_{N-1}\right) \sum_{m=0}^{\infty} p_{m, N}^{a} Q_{m}^{N}\left(z=h_{N}\right) \exp (i k x)
$$

and so on.

Here, $L_{m}$ is the $m$-th order Legendre polynomial. Theoretically, $m$ runs from 0 to $\infty$. In practice, the summation over the polynomials in (8) can be truncated at some finite value $m=M$, at which higher-order terms become negligible.

The forms of $u_{a}(a=1,2,3)$ and $\left(u_{x}, u_{y}, u_{z}\right)$ are chosen in order to automatically incorporate in the calculation the continuity conditions at the interfaces relative to the components of the mechanical displacement. 
Equation (7) is multiplied by $Q_{j}^{1 *}(z), Q_{j}^{2 *}(z), \ldots, Q_{j}^{N *}(z)$, with $j$ running from 0 to $M$, so that integration over $z$ from 0 to $h_{N}$ yields the following $3 N(M+1)$ equations:

$$
\begin{aligned}
& { }^{n} A_{11}^{j, m} p_{m, n}^{1}+{ }^{n} A_{12}^{j, m} p_{m, n}^{2}+{ }^{n} A_{13}^{j, m} p_{m, n}^{3}=-\omega^{2} \cdot{ }^{n} M_{m}^{j} p_{m, n}^{1}, \\
& { }^{n} A_{21}^{j, m} p_{m, n}^{1}+{ }^{n} A_{22}^{j, m} p_{m, n}^{2}+{ }^{n} A_{23}^{j, m} p_{m, n}^{3}=-\omega^{2} \cdot{ }^{n} M_{m}^{j} p_{m, n}^{1}, \\
& { }^{n} A_{31}^{j, m} p_{m, n}^{1}+{ }^{n} A_{32}^{j, m} p_{m, n}^{2}+{ }^{n} A_{33}^{j, m} p_{m, n}^{3}=-\omega^{2} \cdot{ }^{n} M_{m}^{j} p_{m, n}^{1} .
\end{aligned}
$$

where ${ }^{n} A_{\alpha \beta}^{j, m}(\alpha, \beta=1,2,3)$ and ${ }^{n} M_{m}^{j}$ are the elements of a nonsymmetric matrix. They can be easily obtained according to (7).

Equation (9) can be written as

$$
\left[\begin{array}{ccc}
{ }^{n} A_{11}^{j, m} & { }^{n} A_{12}^{j, m} & { }^{n} A_{13}^{j, m} \\
{ }^{n} A_{21}^{j, m} & { }^{n} A_{22}^{j, m} & { }^{n} A_{22}^{j, m} \\
{ }^{n} A_{31}^{j, m} & { }^{n} A_{32}^{j, m} & { }^{n} A_{33}^{j, m}
\end{array}\right]\left\{\begin{array}{l}
p_{m, n}^{1} \\
p_{m, n}^{2} \\
p_{m, n}^{3}
\end{array}\right\}=-\omega^{2}\left[\begin{array}{ccc}
{ }^{n} M_{m}^{j} & 0 & 0 \\
0 & { }^{n} M_{m}^{j} & 0 \\
0 & 0 & { }^{n} M_{m}^{j}
\end{array}\right]\left\{\begin{array}{l}
p_{m, n}^{1} \\
p_{m, n}^{2} \\
p_{m, n}^{3}
\end{array}\right\} .
$$

So, (10) yields a form of the eigenvalue problem. The eigenvalue $\omega^{2}$ gives the angular frequency of the guided wave; eigenvectors $p_{m, n}^{i}(i=1,2,3)$ allow the components of the particle displacement to be calculated. According to $V p h=\omega / k$ and $V g=d \omega / d k$, the phase velocity and group velocity can be obtained. Equation (10) can be solved numerically making use of standard computer programs for the diagonalization of nonsymmetric square matrices. $3 N(M+1)$ eigenmodes are generated from the order- $M$ expansion. Acceptable solutions are those eigenmodes for which convergence is obtained as $M$ is increased. We determine that the eigenvalues obtained are converged when a further increase in the matrix dimension does not result in a significant change in the eigenvalue. We consider $0.5 \%$ as a significant change.

\section{Numerical results}

Based on the foregoing formulations, computer programs have been written using Mathematica to calculate the dispersion curves. We take common ceramic-metal (silicon nitride $(\mathrm{N})$ and steel $(\mathrm{S})$ ) layered plates as examples. Their material properties are listed in Table 1. Because SH waves and Lamb-like waves are independent, we will discuss them respectively.

3.1. Convergence of the method. In order to verify the convergence of the method, the Lamb-like wave and $\mathrm{SH}$ wave dispersion curves for a three-layered plate under an uniform initial stress $Q=20 \mathrm{GPa}$ are calculated for $M=6,7,8$, and 9, as shown in Figure 2. The stacking sequence and thickness $(m)$ of the plate are $\mathrm{N} / \mathrm{S} / \mathrm{N}-1 / 2 / 1$.

\begin{tabular}{|lrrrrrrc|}
\hline Material & $C_{11}$ & $C_{13}$ & $C_{33}$ & $C_{44}$ & $C_{55}$ & $C_{66}$ & $\rho$ \\
Steel & 282 & 113 & 282 & 84 & 84 & 84 & 7.932 \\
Silicon nitride & 380 & 120 & 380 & 130 & 130 & 130 & 2.37 \\
\hline
\end{tabular}

Table 1. The material properties of the layered plate. $C_{i j}$ values are in units of $10^{9} \mathrm{~N} / \mathrm{m}^{2}$, $\rho$ values in $10^{3} \mathrm{~kg} / \mathrm{m}^{3}$. 

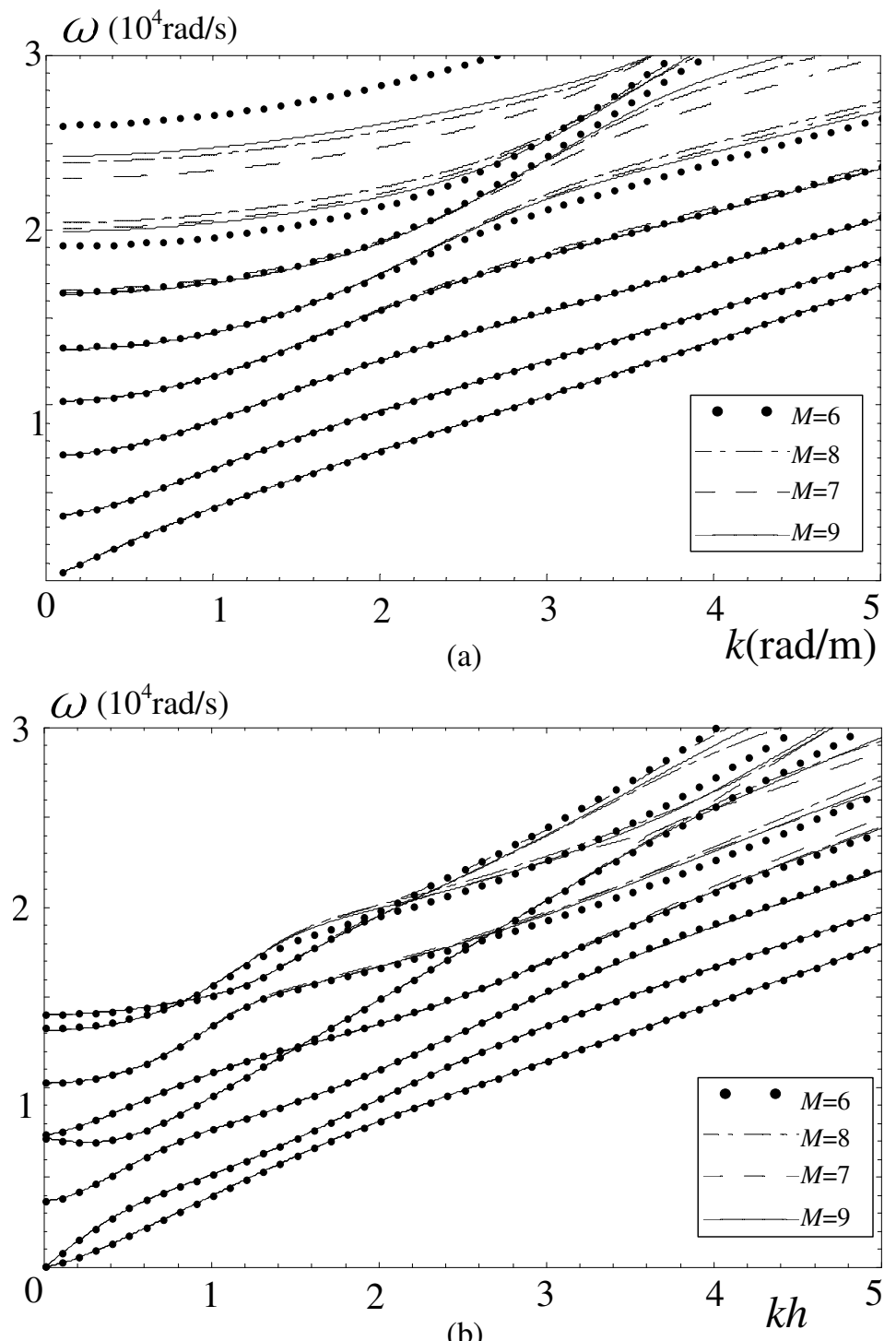

Figure 2. Dispersion curves for the N/S/N-1/2/1 plate under uniform initial stress $Q=$ $20 \mathrm{GPa}$ for various $M$ values. (a) SH waves and (b) Lamb-like waves.

For SH waves, the first three modes are convergent when $M=6$; the first four modes are convergent when $M=7$ and 8 . So, we think that at least the first $M / 2 \mathrm{SH}$ modes are convergent. Similarly, for Lamb-like waves, the first three modes are convergent when $M=6$; the first four modes are convergent when $M=7$ and 8 . So, we believe that at least the first $M / 2$ Lamb-like wave modes are convergent.

For all the numerical calculations presented below, the series expansion is truncated at $M=16$.

3.2. Effects of the initial stresses on Lamb-like waves. Figure 3 shows the phase velocity dispersion curves for the N/S/N-1/2/1 plate under different uniform initial stresses. Figure $3 \mathrm{a}$ is for the plate under initial stresses $Q$ and Figure $3 \mathrm{~b}$ is for the plate under uniform initial stresses $P$, that is, $P_{1}=P_{2}=P_{3}$. 

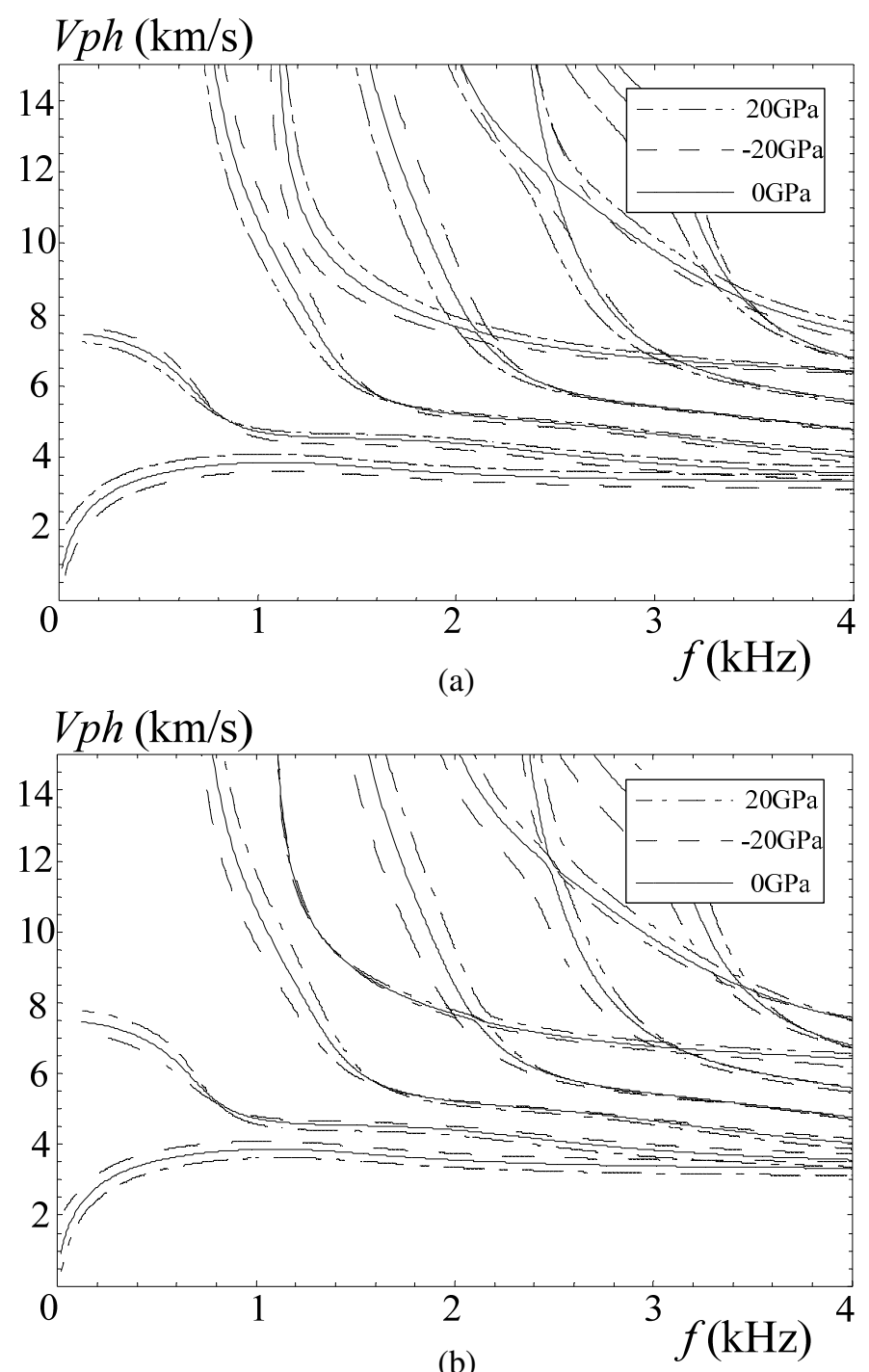

Figure 3. Lamb-like wave phase velocity spectra for the N/S/N-1/2/1 plate, (a) under initial stresses $Q$ and (b) under uniform initial stresses $P$.

According to Figure 3a, a tensile stress in the thickness direction usually makes the wave speed lower at low frequencies (except for the first mode). As the frequency increases, a tensile stress makes the wave speed higher. In many cases, the effect of the tensile stress is contrary to that of the compressive stress. Comparing the two figures, we can see that the effect of the initial stress $P$ is quite different from that of the initial stress $Q$. In many cases, the effect of the initial stress $P$ is almost contrary to that of the initial stress $Q$.

Figure 3 reflects the relation between the effect of the initial stress and frequency. We observe the relation between the frequency (or wave speed) and the magnitudes of initial stress $Q$ and $P$, as shown in Figures $4 \mathrm{a}$ and $4 \mathrm{~b}$, which show the frequency versus initial stress $Q$ and $P$ at $k=1 \mathrm{rad} / \mathrm{m}$. In fact, 

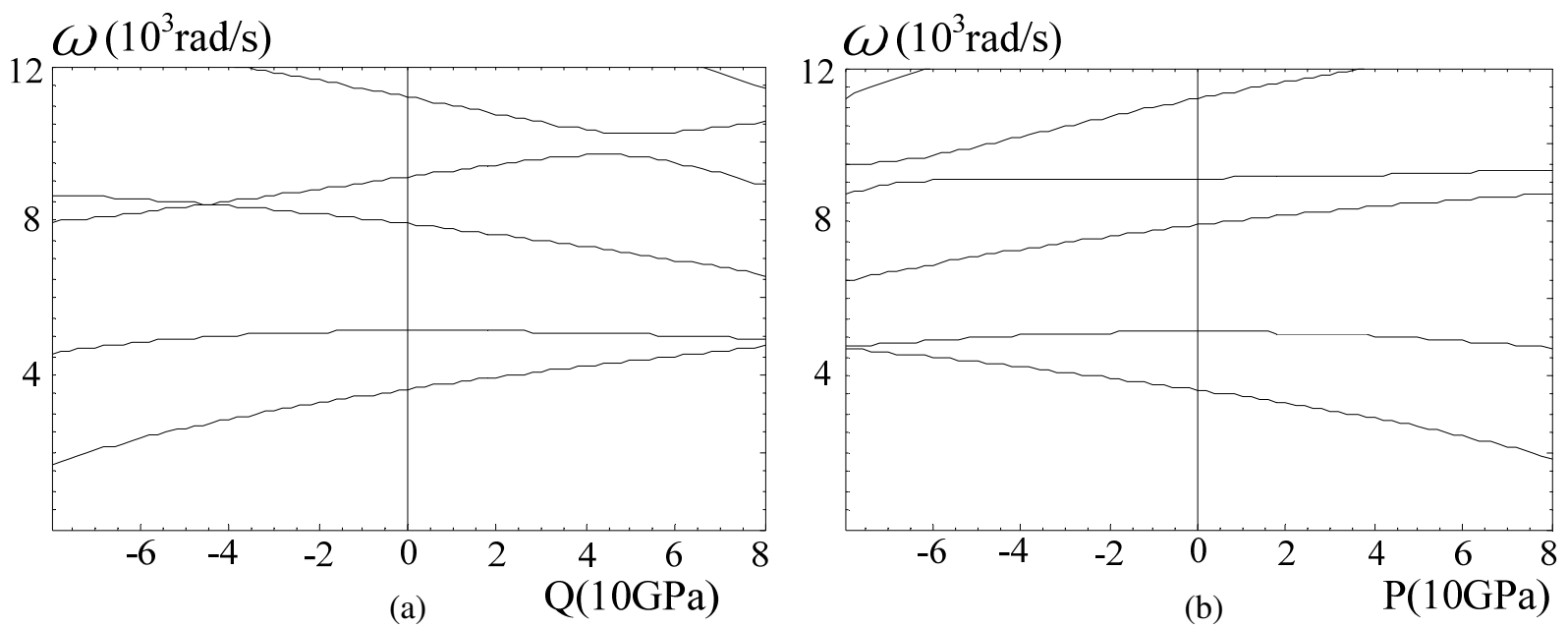

Figure 4. Lamb-like wave frequency curves versus initial stresses for the $\mathrm{N} / \mathrm{S} / \mathrm{N}-1 / 2 / 1$ plate at $k=1 \mathrm{rad} / \mathrm{m}$, with (a) uniform initial stresses $Q$ and (b) uniform initial stresses $P$.

these two figures also reflect the relation between the wave speed and initial stress because of the fixed wave number. It can be seen that with the increase of the initial stresses in both directions, the frequency variation is not linear and the varying trends of each mode are different. The effects of the compressive stress and tensile stress do not always contrast. For instance, for mode 2, the highest frequency is at about the initial stress $Q=0$ and $P=0$; both compressive stress and tensile stress make the wave speed and frequency lower.

In the above examples, the initial stresses in the wave-propagation direction are uniform, that is, $P_{1}=P_{2}=P_{3}$. Now we illustrate the differences in the effects of $P_{1}$ and $P_{2}$. In order to facilitate the comparison, we take an equal-thickness layered plate, N/N/N-1/1/1, as an example. We calculate three cases: $P_{1}=P_{2}=P_{3}=0 ; P_{1}=-80 \mathrm{GPa}, P_{2}=P_{3}=0$; and $P_{2}=-80 \mathrm{GPa}, P_{1}=P_{3}=0$. The obtained dispersion curves and the frequency-stress curves are shown in Figures 5 and 6 . It can be seen that the effects of $P_{1}$ and $P_{2}$ are different. We cannot conclude that the effect of one is stronger than that of another one. For different modes, the extents of their influence are different, and vary as the frequency increases.

3.3. Effects of the initial stresses on $\mathbf{S H}$ waves. This section concerns the effects of the initial stresses on $\mathrm{SH}$ waves. Figures 7 and 8 show the dispersion curves for the N/S/N-1/2/1 plate under different uniform initial stresses $P$ and $Q$. It can be seen that the effects of initial stresses are very regular. For the case of initial stress in the wave-propagation direction, compressive stress always makes the wave speed lower but tensile stress makes the wave speed higher. At small wavenumbers, the effect is weak. As the wavenumber increases, the effect becomes stronger. The effect of initial stresses $Q$ is quite different from that of initial stresses $P$ except that compressive stress still makes the wave speed lower. For a plate with the initial stress in the thickness direction, the effect of initial stresses does not change significantly as the wavenumber increases, but it becomes stronger as the mode order increases and it is very weak on the first mode. 


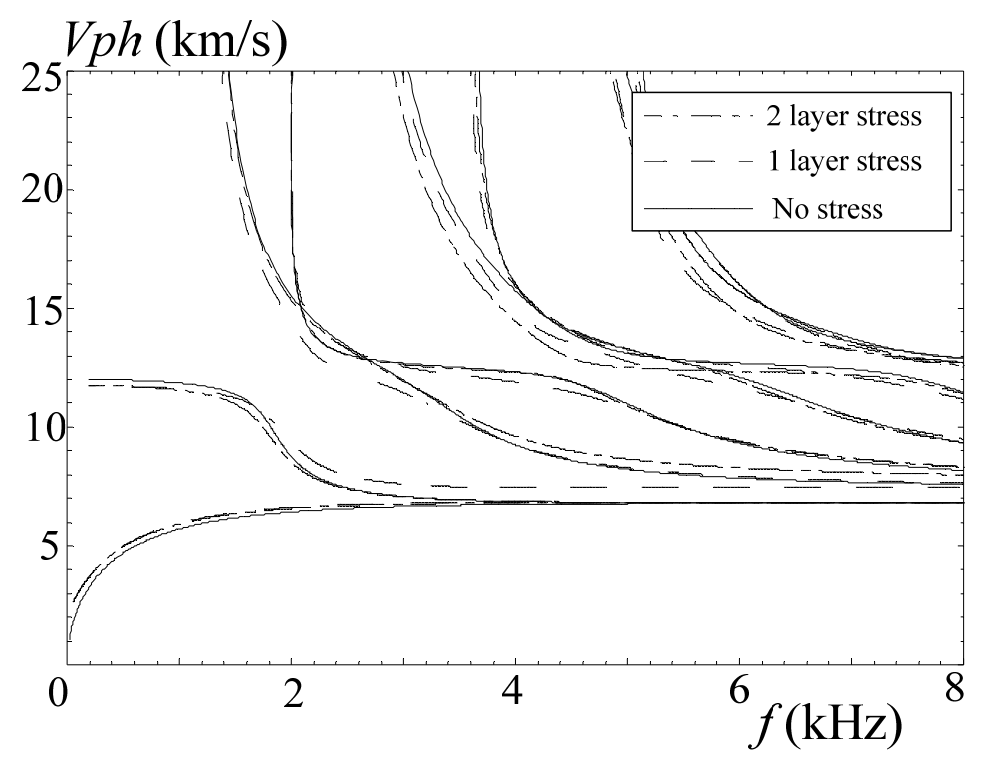

Figure 5. Lamb-like wave phase velocity spectra for the N/N/N-1/1/1 plate under initial stresses $P ; 1$ layer stress represents $P_{1}=-80 \mathrm{GPa}, P_{2}=P_{3}=0$; and 2 layer stress represents $P_{2}=-80 \mathrm{GPa}, P_{1}=P_{3}=0$.
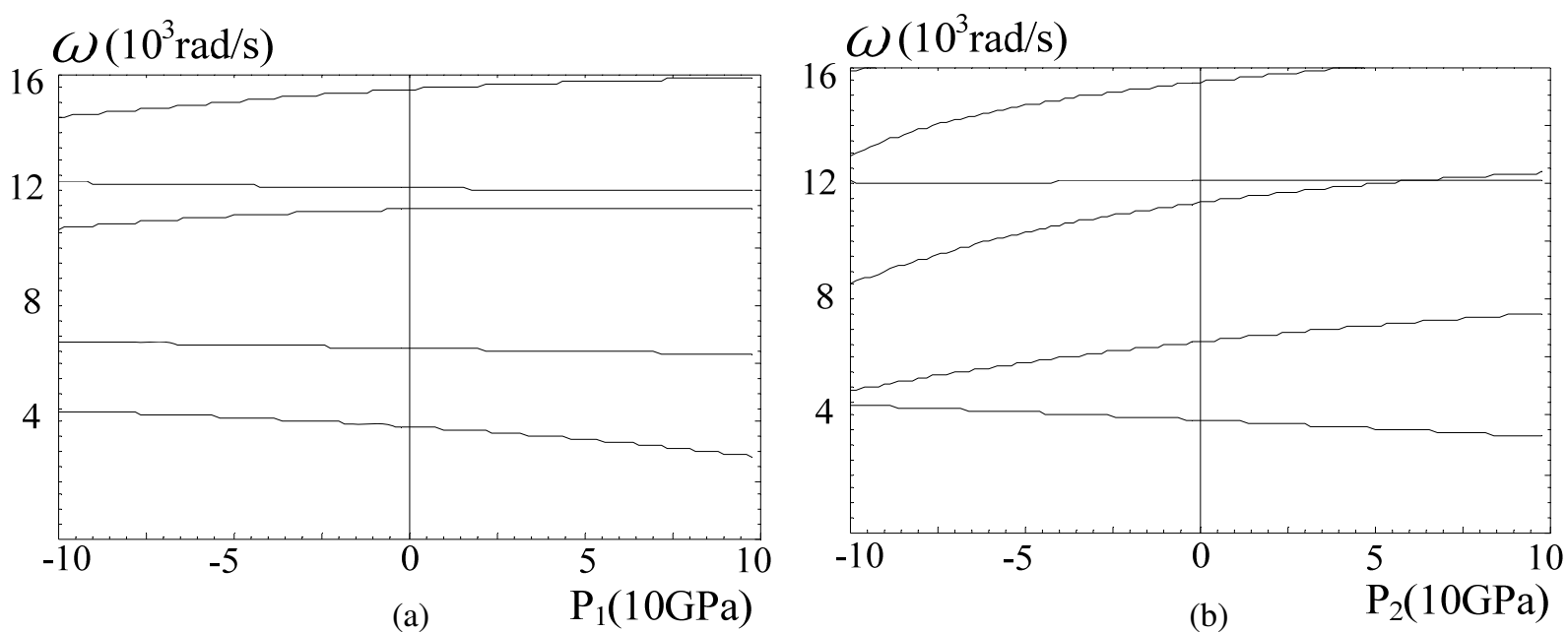

Figure 6. Lamb-like wave frequency curves versus initial stresses for the N/N/N-1/1/1 plate at $k=1 \mathrm{rad} / \mathrm{m}$, with (a) initial stresses $P_{1}$ and (b) initial stresses $P_{2}$.

Figure 9 illustrates the curves of frequency with initial stresses $P$ and $Q$ for the N/S/N-1/2/1 plate at $k=1 \mathrm{rad} / \mathrm{m}$. It can be seen that with the increase of the initial stresses in both directions, the frequencies of all modes approximately linearly lower. The effect of compressive stress is entirely contrary to that of tensile stress. For the initial stresses in the wave-propagation direction, the slopes of the frequency curves 

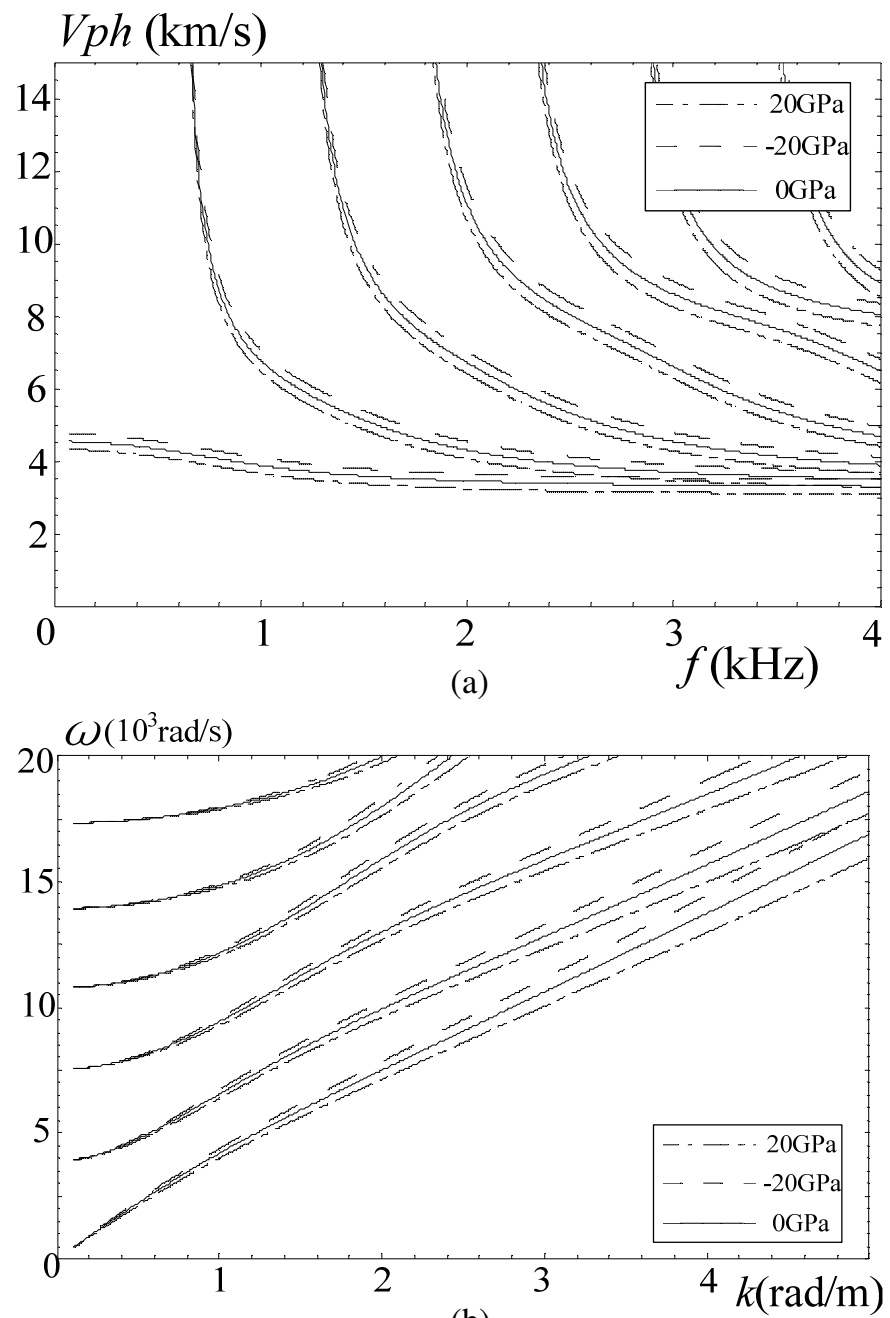

(b)

Figure 7. $\mathrm{SH}$ wave dispersion curves for the N/S/N-1/2/1 plate under uniform initial stresses $P$; (a) phase velocity spectra and (b) frequency spectra.

for all modes are similar. For the initial stresses in the thickness direction, the slope of the frequency curve for the first mode is very small. As the mode order increases, the slope becomes larger.

\section{Conclusions}

Following [Biot 1965], guided wave propagation in layered plates under initial stresses in the thickness and wave-propagation directions are investigated. The Legendre polynomial series method is used to solve the coupled wave equations. The effects of the initial stresses on the dispersion curves are discussed. Based on the calculated results, the following conclusions can be drawn:

(1) The effect of the initial stress on Lamb-like waves is quite different from that on the SH waves. The effect on SH waves is very regular. 

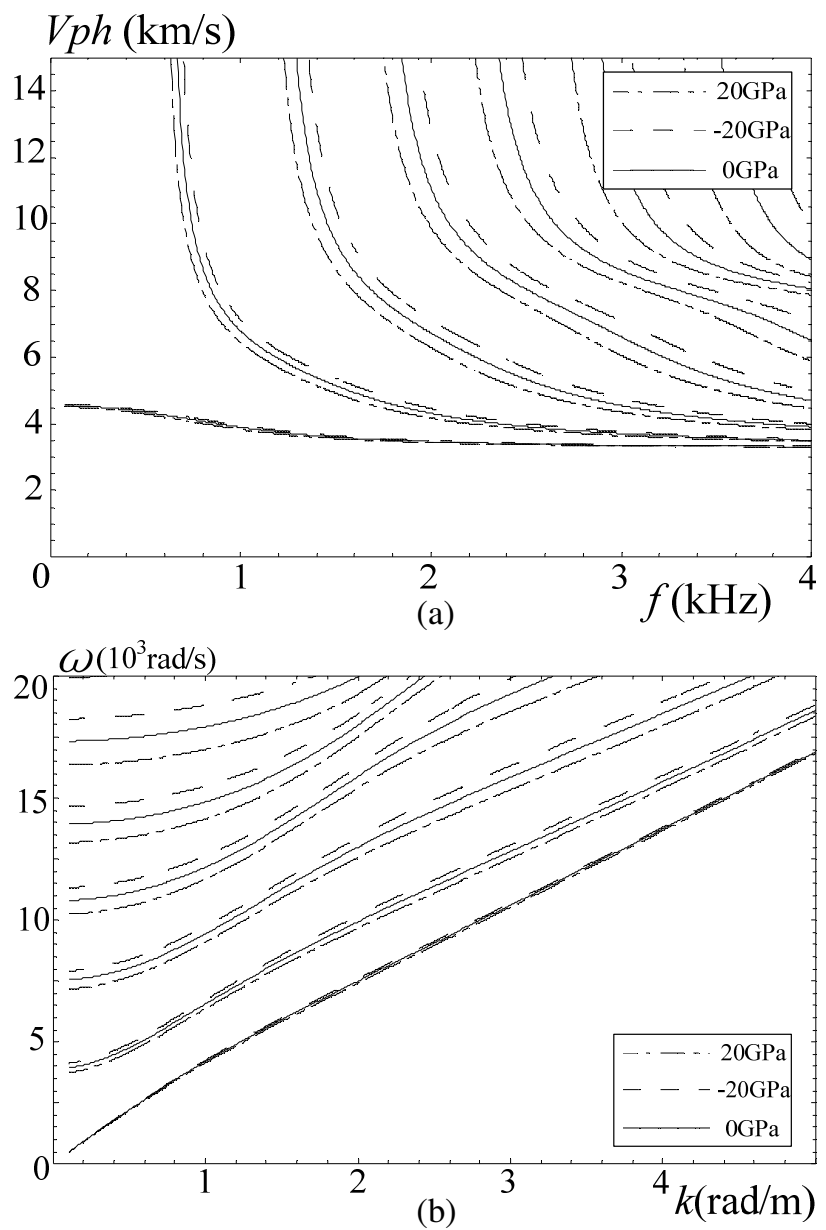

Figure 8. $\mathrm{SH}$ wave dispersion curves for the $\mathrm{N} / \mathrm{S} / \mathrm{N}-1 / 2 / 1$ plate under initial stresses $Q$; (a) phase velocity spectra and (b) frequency spectra.
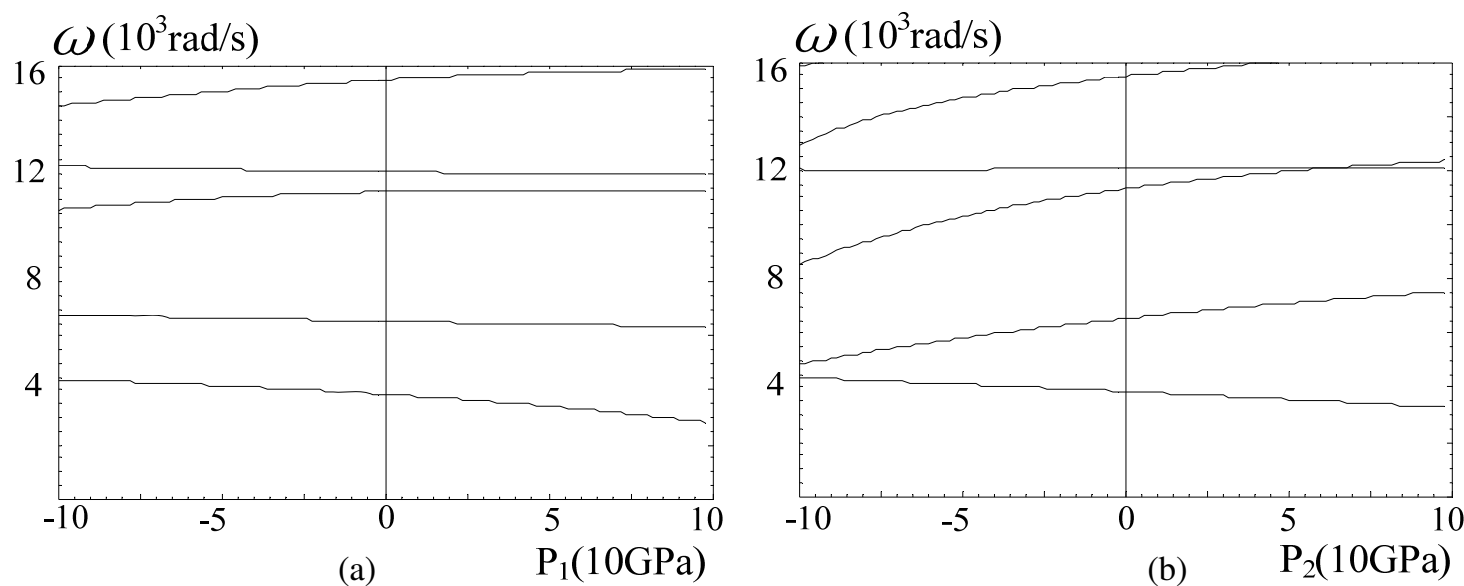

Figure 9. $\mathrm{SH}$ wave frequency curves versus initial stresses for the $\mathrm{N} / \mathrm{S} / \mathrm{N}-1 / 2 / 1$ plate at $k=1 \mathrm{rad} / \mathrm{m}$, with (a) uniform initial stresses $Q$ and (b) uniform initial stresses $P$. 
(2) The effect of the compressive stress on Lamb-like waves is not always contrary to that of the tensile stress. But the effect of the compressive stress on SH waves is almost entirely contrary to that of the tensile stress.

(3) For Lamb-like waves, the effect of the initial stress in the thickness direction usually is contrary to that of the initial stress in the wave-propagation direction. However, for SH waves, the effects of initial stresses in the two directions do not contrast, but their influential patterns are different.

(4) For Lamb-like waves, with the increase of the initial stresses in both directions, the frequency variation is not linear and the varying trends of every mode are different. For SH waves, the curves of the frequency versus the stresses are approximately linear for all modes.

\section{Acknowledgment}

The work was supported by the National Natural Science Foundation of China (No. 11272115). Jiangong Yu gratefully acknowledges the support of the Alexander von Humboldt-Foundation for conducting research work at the university of Siegen, Germany.

\section{References}

[Abd-Alla 1999] A. M. Abd-Alla, "The effect of initial stress and orthotropy on the propagation waves in a hollow cylinder", Appl. Math. Comput. 106:2-3 (1999), 237-244.

[Abd-Alla and Ahmed 1999] A. M. Abd-Alla and S. M. Ahmed, "Propagation of Love waves in a non-homogeneous orthotropic elastic layer under initial stress overlying semi-infinite medium”, Appl. Math. Comput. 106:2-3 (1999), $265-275$.

[Akbarov and Guliev 2009] S. D. Akbarov and M. S. Guliev, "Axisymmetric longitudinal wave propagation in a finite prestrained compound circular cylinder made from compressible materials", CMES Comput. Model. Eng. Sci. 39:2 (2009), 155177.

[Akbarov and Guliev 2010] S. D. Akbarov and M. S. Guliev, "The influence of the finite initial strains on the axisymmetric wave dispersion in a circular cylinder embedded in a compressible elastic medium", International Journal of Mechanical Sciences 52:1 (2010), 89-95.

[Akbarov et al. 2008] S. D. Akbarov, A. D. Zamanov, and E. R. Agasiyev, "On the propagation of Lamb waves in a sandwich plate made of compressible materials with finite initial strains", Mechanics of Composite Materials 44:2 (2008), 155-164.

[Akbarov et al. 2011a] S. D. Akbarov, E. R. Agasiyev, and A. D. Zamanov, "Wave propagation in a pre-strained compressible elastic sandwich plate", European Journal of Mechanics A/Solids 30:3 (2011), 409-422.

[Akbarov et al. 2011b] S. D. Akbarov, T. Kepceler, and M. M. Egilmez, "Torsional wave dispersion in a finitely pre-strained hollow sandwich circular cylinder", Journal of Sound and Vibration 330:18-19 (2011), 4519-4537.

[Bigoni et al. 2008] D. Bigoni, M. Gei, and A. B. Movchan, "Dynamics of a prestressed stiff layer on an elastic half space: filtering and band gap characteristics of periodic structural models derived from long-wave asymptotics", J. Mech. Phys. Solids 56:7 (2008), 2494-2520.

[Biot 1965] M. A. Biot, Mechanics of incremental deformations: theory of elasticity and viscoelasticity of initially stressed solids and fluids, including thermodynamic foundations and applications to finite strain, Wiley, New York, 1965.

[Chen and Wright 1966] W. T. Chen and T. W. Wright, "Frequency equations for wave propagation in an initially stressed circular cylinder", The Journal of the Acoustical Society of America 39:5A (1966), 847-848.

[Datta and Hunsinger 1978] S. Datta and B. J. Hunsinger, "Analysis of surface waves using orthogonal functions", Journal of Applied Physics 49:2 (1978), 475-479.

[Du et al. 2007] J. Du, X. Jin, and J. Wang, "Love wave propagation in layered magneto-electro-elastic structures with initial stress", Acta Mechanica 192:1-4 (2007), 169-189. 
[El-Naggar et al. 1994] A. M. El-Naggar, A. M. Abd-Alla, and S. M. Ahmed, "Rayleigh waves in a magnetoelastic initially stressed conducting medium with the gravity field", Bull. Calcutta Math. Soc. 86:3 (1994), 51-56.

[Garg 2007] N. Garg, "Effect of initial stress on harmonic plane homogeneous waves in viscoelastic anisotropic media", $J$. Sound Vib. 303:3-5 (2007), 515-525.

[Gei 2008] M. Gei, "Elastic waves guided by a material interface”, Eur. J. Mech. A Solids 27:3 (2008), 328-345.

[Gei et al. 2004] M. Gei, D. Bigoni, and G. Franceschini, "Thermoelastic small-amplitude wave propagation in nonlinear elastic multilayers", Mathematics and Mechanics of Solids 9:5 (2004), 555-568.

[Gupta et al. 2012] S. Gupta, D. K. Majhi, S. Kundu, and S. K. Vishwakarma, "Propagation of torsional surface waves in a homogeneous layer of finite thickness over an initially stressed heterogeneous half-space", Appl. Math. Comput. 218:9 (2012), $5655-5664$.

[Kayestha et al. 2010] P. Kayestha, A. C. Wijeyewickrema, and K. Kishimoto, "Time-harmonic wave propagation in a prestressed compressible elastic bi-material laminate", Eur. J. Mech. A Solids 29:2 (2010), 143-151.

[Murdoch 1977] A. I. Murdoch, "The effect of interfacial stress on the propagation of Stoneley waves", Journal of Sound and Vibration 50:1 (1977), 1-11.

[Ogden and Sotiropoulos 1995] R. W. Ogden and D. A. Sotiropoulos, "On interfacial waves in pre-stressed layered incompressible elastic solids", Proc. Roy. Soc. London Ser. A 450:1939 (1995), 319-341.

[Qian et al. 2004] Z. Qian, F. Jin, Z. Wang, and K. Kishimoto, "Love waves propagation in a piezoelectric layered structure with initial stresses", Acta Mech. 171:1-2 (2004), 41-57.

[Rogerson and Sandiford 1996] G. A. Rogerson and K. J. Sandiford, "On small amplitude vibrations of pre-stressed laminates", International Journal of Engineering Science 34:8 (1996), 853-872.

[Selim and Ahmed 2006] M. M. Selim and M. K. Ahmed, "Plane strain deformation of an initially stressed orthotropic elastic medium”, Appl. Math. Comput. 175:1 (2006), 221-237.

[Shmuel et al. 2012] G. Shmuel, M. Gei, and G. deBotton, "The Rayleigh-Lamb wave propagation in dielectric elastomer layers subjected to large deformations", International Journal of Non-linear Mechanics 47:2 (2012), 307-316.

[Wijeyewickrema and Leungvichcharoen 2009] A. C. Wijeyewickrema and S. Leungvichcharoen, "Wave propagation in prestressed imperfectly bonded compressible elastic layered composites", Mechanics of Materials 41:10 (2009), 1192-1203.

[Zamanov and Agasiyev 2011] A. D. Zamanov and E. R. Agasiyev, "Dispersion of lamb waves in a three-layer plate made from compressible materials with finite initial deformations", Mechanics of Composite Materials 46:6 (2011), 583-592.

Received 26 Oct 2012. Revised 31 Jan 2013. Accepted 31 Jan 2013.

JIANGONG YU: yu@emails.bjut.edu.cn

School of Mechanical and Power Engineering, Henan Polytechnic University, Jiaozuo, 454003 Henan, China

SHENLEI LI: $982368392 @ q q . c o m$

School of Mechanical and Power Engineering, Henan Polytechnic University, Jiaozuo, 454003 Henan, China 


\title{
JOURNAL OF MECHANICS OF MATERIALS AND STRUCTURES
}

\author{
msp.org/jomms
}

\author{
Founded by Charles R. Steele and Marie-Louise Steele
}

EDITORIAL BOARD

ADAIR R. AGUIAR

KATIA BERTOLDI

University of São Paulo at São Carlos, Brazil

DAVIDE BIGONI

Harvard University, USA

IWONA JASIUK

University of Trento, Italy

Thomas J. PENCE

University of Illinois at Urbana-Champaign, USA

YASUHIDE SHINDO

Michigan State University, USA

DAVID STEIGMANN

Tohoku University, Japan

University of California at Berkeley

\section{ADVISORY BOARD}

J. P. CARTER University of Sydney, Australia

R. M. Christensen Stanford University, USA

G. M. L. GLADWELL University of Waterloo, Canada

D. H. Hodges Georgia Institute of Technology, USA

J. HUTCHINSON Harvard University, USA

C. HwU National Cheng Kung University, Taiwan

B. L. KARIHALOO University of Wales, UK

Y. Y. KIM Seoul National University, Republic of Korea

Z. Mroz Academy of Science, Poland

D. PAMPlonA Universidade Católica do Rio de Janeiro, Brazil

M. B. RubiN Technion, Haifa, Israel

A. N. SHUPIKOV Ukrainian Academy of Sciences, Ukraine

T. TARNAI University Budapest, Hungary

F. Y. M. WAN University of California, Irvine, USA

P. WRIGGERS Universität Hannover, Germany

W. YANG Tsinghua University, China

F. ZIEGLER Technische Universität Wien, Austria

PRODUCTION production@msp.org

SILVIO LEVY Scientific Editor

Cover photo: Mando Gomez, www.mandolux.com

See msp.org/jomms for submission guidelines.

JoMMS (ISSN 1559-3959) at Mathematical Sciences Publishers, 798 Evans Hall \#6840, c/o University of California, Berkeley, CA 94720-3840, is published in 10 issues a year. The subscription price for 2013 is US \$555/year for the electronic version, and $\$ 705 /$ year (+\$60, if shipping outside the US) for print and electronic. Subscriptions, requests for back issues, and changes of address should be sent to MSP.

JoMMS peer-review and production is managed by EditFLOW ${ }^{\circledR}$ from Mathematical Sciences Publishers.

PUBLISHED BY

mathematical sciences publishers

nonprofit scientific publishing

http://msp.org/

(C) 2013 Mathematical Sciences Publishers 


\section{Journal of Mechanics of Materials and Structures}

\section{Volume 8, No.:2-4 April-June 2013}

A diffuse cohesive energy approach to fracture and plasticity: the one-dimensional case

Gianpietro Del Piero, Giovanni Lancioni and Riccardo March 109

Continuum deployable shells made of thin plates

Vladimir A. GracheV and Yuriy S. NeUSTAdT

Self-folding of a slender microbeam and thin film: an elastica model

JIANLIN LIU and JUNG HoON LEE 169

Dispersion of guided waves in initially stressed layered plates

JIANGONG YU and SHENLEI LI 185

Empirical models for predicting protective properties of concrete shields against

high-speed impact

GABI BEN-Dor, ANATOLY DUBinsky and Tov ELPERIN

Transient response of a general anisotropic solid to dislocation growth: alternative formulation LOUIS MILTON BROCK 